\title{
Prostate Cancer Incidence Across Stage, NCCN Risk Groups, and Age Before and After USPSTF Grade D Recommendations Against Prostate-Specific Antigen Screening in 2012
}

\author{
Santino S. Butler (ID) 1; Vinayak Muralidhar, MD, MSc'; Shuang G. Zhao, MD²; Nina N. Sanford, MD³; Idalid Franco, MD, MPH'; \\ Zoe H. Fullerton (iD '; Janice Chavez, MSW'; Anthony V. D’Amico, MD'; Felix Y. Feng, MD ${ }^{4}$; Timothy R. Rebbeck, PhD ${ }^{1,5}$; \\ Paul L. Nguyen, MD'; and Brandon A. Mahal, MD iD 1
}

\begin{abstract}
BACKGROUND: We sought to determine the extent to which US Preventive Services Task Force (USPSTF) 2012 Grade D recommendations against prostate-specific antigen screening may have impacted recent prostate cancer disease incidence patterns in the United States across stage, National Comprehensive Cancer Network (NCCN) risk groups, and age groups. METHODS: SEER*Stat version 8.3.4 was used to calculate annual prostate cancer incidence rates from 2010 to 2015 for men aged $\geq 50$ years according to American Joint Committee on Cancer stage at diagnosis (localized vs metastatic), NCCN risk group (low vs unfavorable [intermediate or high-risk]), and age group (50-74 years vs $\geq 75$ years). Age-adjusted incidences per 100,000 persons with corresponding year-by-year incidence ratios (IRs) were calculated using the 2000 US Census population. RESULTS: From 2010 to 2015, the incidence (per 100,000 persons) of localized prostate cancer decreased from 195.4 to $131.9\left(P_{\text {trend }}<.001\right)$ and from 189.0 to $123.4\left(P_{\text {trend }}<.001\right)$ among men aged $50-74$ and $\geq 75$ years, respectively. The largest relative year-by-year decline occurred between 2011 and 2012 in NCCN low-risk disease (IR, 0.77 $[0.75-0.79, P<.0001]$ and IR $0.68[0.62-0.74, P<.0001]$ for men aged $50-74$ and $\geq 75$ years, respectively). From 2010-2015, the incidence of metastatic disease increased from 6.2 to $7.1\left(P_{\text {trend }}<.001\right)$ and from 16.8 to $22.6\left(P_{\text {trend }}<.001\right)$ among men aged $50-74$ and $\geq 75$ years, respectively. CONCLUSIONS: This report illustrates recent prostate cancer "reverse migration" away from indolent disease and toward more aggressive disease beginning in 2012. The incidence of localized disease declined across age groups from 2012 to 2015 , with the greatest relative declines occurring in low-risk disease. Additionally, the incidence of distant metastatic disease increased gradually throughout the study period. Cancer 2020;126:717-724. @ 2019 American Cancer Society.
\end{abstract}

KEYWORDS: prostatic neoplasms, PSA screening, incidence, prostate cancer.

\section{INTRODUCTION}

The prostate-specific antigen (PSA) screening era led to a drastic increase in prostate cancer detection, along with a migration toward more indolent disease at diagnosis. ${ }^{1}$ However, following an October $2011 \mathrm{draft}$ statement, the US Preventive Services Task Force (USPSTF) made a formal Grade D recommendation against PSA screening for all men in 2012 (similar to 2008 Grade D recommendations for men aged $\geq 75$ years) ${ }^{2,3}$ given questions about screening efficacy and concern that screening may lead to overdetection and treatment of indolent disease. ${ }^{4,5}$ However, consensus on an optimal screening paradigm remains sparse, and some specialists and cancer organizations continue to favor routine PSA screening for younger, healthy men. ${ }^{6}$ Moreover, longer follow-up in PSA screening trials has demonstrated the increasing efficacy of PSA screening over time given the indolent nature of prostate cancer. ${ }^{7,8}$ Therefore, in 2018 the USPSTF made a Grade $\mathrm{C}$ recommendation supporting individualized PSA screening decisions for men aged 55-69 years. ${ }^{8}$

Using contemporary population-based data, we sought to determine the extent to which 2012 USPSTF recommendations against PSA screening may have impacted recent prostate cancer incidence patterns in the United States across stage, National Comprehensive Cancer Network $(\mathrm{NCCN})^{9}$ risk groups, and age groups.

\section{MATERIALS AND METHODS}

To illustrate trends in incidence patterns over time, SEER*Stat version 8.3.4 was used to calculate annual age-adjusted incidence rates of prostate cancer from 2010 to 2015 among men aged $\geq 50$ years in the Surveillance, Epidemiology,

Corresponding Author: Brandon A. Mahal, MD, Department of Radiation Oncology, 75 Francis Street, Boston, MA 02115 (brandon_mahal@dfci.harvard.edu).

${ }^{1}$ Dana-Farber Cancer Institute/Brigham and Women's Hospital, Harvard Medical School, Boston, Massachusetts; ${ }^{2}$ Department of Radiation Oncology, University of Michigan, Ann Arbor, Michigan; ${ }^{3}$ Department of Radiation Oncology, University of Texas Southwestern Medical Center, Dallas, Texas; ${ }^{4}$ University of California, San Francisco, California; ${ }^{5}$ Harvard T.H. Chan School of Public Health, Boston, Massachusetts

See editorial on pages 694-6, this issue.

Additional supporting information may be found in the online version of this article.

DOI: 10.1002/cncr.32604, Received: February 12, 2019; Revised: July 15, 2019; Accepted: August 14, 2019, Published online December 3, 2019 in Wiley Online Library (wileyonlinelibrary.com) 
and End-Results (SEER) 18 Regs Custom Data (with additional treatment fields), Nov 2017 Sub (20102015) Database. ${ }^{10}$ The SEER 18 program collects and publishes cancer incidence data from 18 populationbased cancer registries covering approximately $27.8 \%$ of the US population (based on the 2010 Census).

Trends in rates were compared using Cuzick's test. Incidence rates were calculated by stage at diagnosis (American Joint Committee on Cancer [AJCC] 7th Edition localized disease [T1-T4 N0M0] versus AJCC 7th Edition distant metastatic disease [M1]), and stratified by age group (50-74 years versus $\geq 75$ years) based on USPSTF PSA screening recommendations. ${ }^{2,3}$ With the recent inclusion of validated and qualityassured PSA data in SEER, ${ }^{11}$ incidence rates were also calculated for localized NCCN-defined risk groups (low [PSA $<10 \mathrm{ng} / \mathrm{mL}$ and $\mathrm{cT} 1-2 \mathrm{a}$ and Gleason $\leq 6$ ] vs unfavorable [intermediate or high-risk] disease [PSA $>10 \mathrm{ng} / \mathrm{mL}$ or cT2b-T4 or Gleason $7-10])^{9}$ in patients with known PSA, clinical tumor stage, and clinical Gleason score. The patient selection included all years for which clinical information on NCCN risk factors is available in SEER (2010-2015).

Age-adjusted incidence rates and corresponding 95\% confidence intervals (CIs) were expressed per 100,000 persons using the 2000 US Census standard population, with adjustments for delays in reporting. To compare incidence rate changes across consecutive years, the Tiwari method was applied to define yearby-year incidence ratios (IRs) with associated 95\% CIs and $P$ values, using the earlier year as reference (ie, 2010 [referent] vs 2011). ${ }^{12,13}$

$P$ values were 2 -sided with $\alpha=.05$. The data are publicly available and deidentified and are therefore considered exempt by the Dana-Farber/Harvard Cancer Center institutional review board.

\section{RESULTS}

From 2010 to 2015, there was a decrease in the annual incidence (per 100,000 persons) of localized prostate cancer from 195.4 to $131.9\left(P_{\text {trend }}<.001\right)$ and from 189.0 to $123.4\left(P_{\text {trend }}<.001\right)$ among men aged $50-74$ years and $\geq 75$ years, respectively (Fig. 1 , Supporting Table 1 ). Conversely, there was an increase in the incidence of metastatic disease from 6.2 to $7.1\left(P_{\text {trend }}<.001\right)$ and from 16.8 to $22.6\left(P_{\text {trend }}<.001\right)$ among men aged $50-74$ years and $\geq 75$ years, respectively (Fig. 1 , Supporting Table 1 ). There was also a decrease in the annual incidence of both localized NCCN low-risk and unfavorable (intermediate/high)risk localized prostate cancer from 60.6 to 31.4 and from
104.2 to 84.3 , respectively, among men aged $50-74$ years. Among men aged $\geq 75$ years, incidence rates decreased similarly from 26.1 to 11.6 and from 134.5 to 94.7, respectively (all $P_{\text {trend }}<.001$ ) (Fig. 1, Supporting Table 1).

The largest relative year-by-year decline in incidence of localized disease occurred between 2011 and 2012, regardless of age or risk group (Fig. 2, Supporting Table 2). The incidence of low-risk disease began to decline in 2012 across age groups, although the decline was relatively greater among men aged $\geq 75$ years (incidence ratio [IR], 0.68 [95\% CI, 0.62-0.74]) compared with men aged 50-74 years (IR, 0.77 [95\% CI, 0.75-0.79]). Notably, the subgroup with the largest relative year-by-year decline in incidence observed in this study occurred between 2011 and 2012 in low-risk disease among men aged $\geq 75$ years (Fig. 2, Supporting Table 2). The incidence of low-risk disease declined in each consecutive year from 2012 to 2015 among men aged 50-74 years $(P<.0001$ for all IRs [Fig. 2, Supporting Table 2]). For men aged $\geq 75$ years however, incidence rates declined from 2012 to 2014 before stabilizing from 2014 to 2015 (Fig. 2, Supporting Table 2). Overall, from 2010 to 2015 there was a greater absolute decline in the incidence of low-risk disease among men aged 50-74 years (29.2 fewer cases per 100,000 men) compared with men aged $\geq 75$ years (14.5 fewer cases per 100,000 men) (Fig. 1, Supporting Table 1).

The incidence of unfavorable (intermediate/high)risk disease also began to decline between 2011 and 2012 across age groups, although the declines were relatively smaller compared with low-risk disease. Declining incidence rates in 2012 were again greater among men aged $\geq 75$ years (IR, 0.78 [95\% CI, 0.75-0.81]) compared with men aged 50-74 years (IR, 0.86 [95\% CI, 0.84-0.88]). Notably, incidence rates declined in consecutive years until $2014(P<.01$ for all IRs across age groups) before increasing from 2014 to 2015 , from 79.3 to 84.3 (IR, 1.06 [95\% CI, 1.04-1.08]) and from 89.0 to 94.7 (IR, 1.06 [95\% CI, 1.02-1.11]) among men aged 50-74 and $\geq 75$ years, respectively.

Metastatic disease incidence increased incrementally such that consecutive year-by-year IRs remained comparable for both age groups, with the exception of increases among men aged $\geq 75$ years between 2011 and 2012 (IR, 1.14 [95\% CI, 1.04-1.26]) and between 2014 and 2015 (IR, 1.13 [95\% CI, 1.04-1.23]). However, when comparing 2015 with 2010, there were significant increases in metastatic disease across age groups (IR, 1.14 [95\% CI, 1.06-1.23] and IR, 1.34 [95\% CI, 1.23-1.47] for men aged 50-74 years and $\geq 75$ years, respectively). 

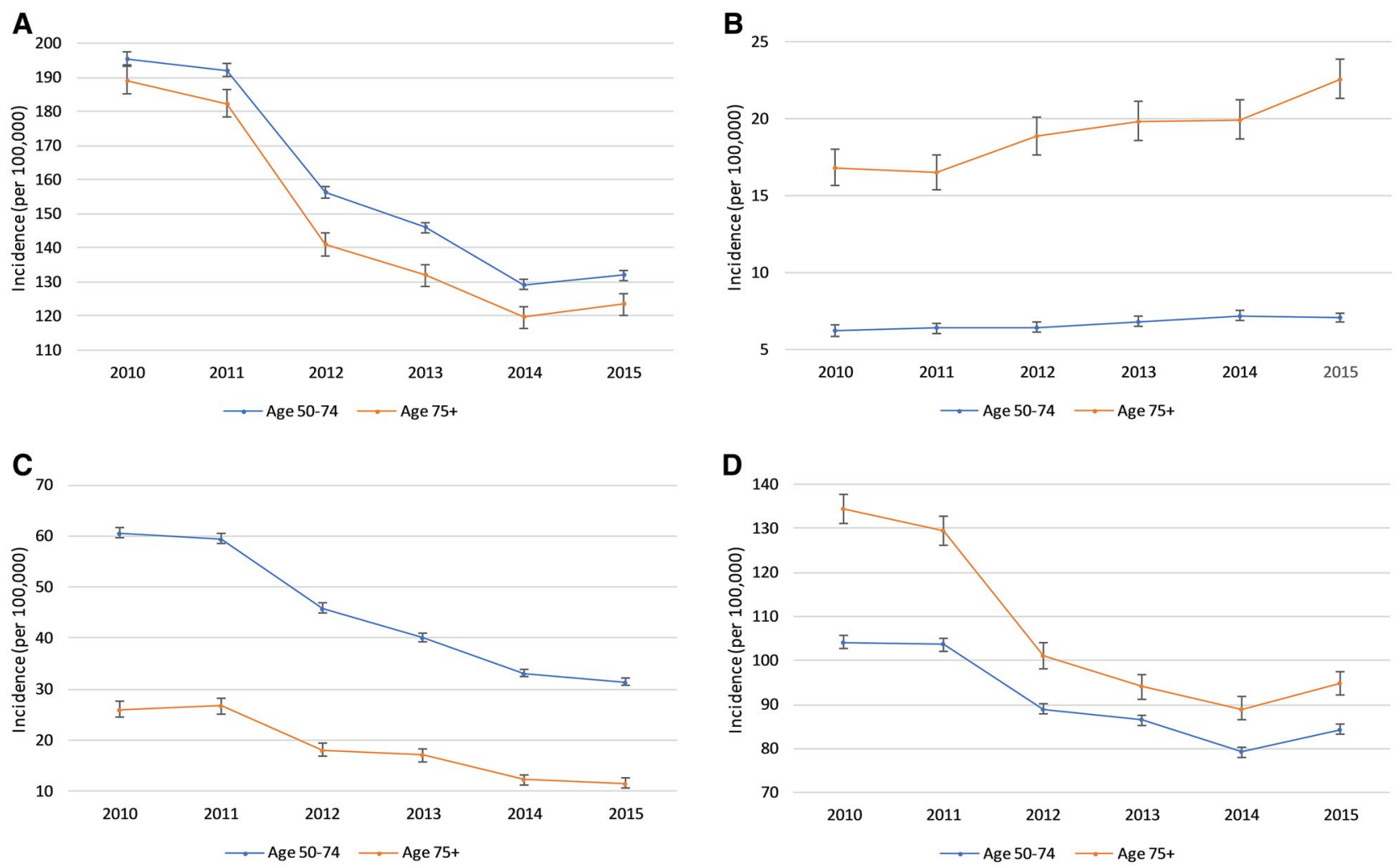

Figure 1. Age-adjusted annual incidence rates (per 100,000 persons using the 2000 US Census standard population) of prostate cancer diagnoses in the SEER*Stat U.S. Database stratified by age group ( $\geq 75$ years vs 50-74 years) for (A) localized disease (NOMO), (B) metastatic disease (M1), (C) National Comprehensive Cancer Network (NCCN) low-risk localized disease, and (D) NCCN unfavorable (intermediate/high)-risk localized disease. Error bars represent 95\% Cls. The unknown NCCN risk group accounts for the remaining incidence of localized disease.

\section{DISCUSSION}

Utilizing contemporary population-based incidence data, this report illustrates recent prostate cancer "reverse migration" away from indolent and toward more aggressive presentation in the United States following USPSTF Grade D recommendations against PSA screening in 2012 (with a draft statement released in October 2011 and an official statement released in May 2012). The data demonstrate a significant decline in the incidence of localized disease across age groups from 2010 to 2015 , with the greatest declines observed between 2011 and 2012. Notably, the greatest relative declines in incidence rates were observed in NCCN lowrisk localized disease, where rates continued to decline throughout the study period, whereas the incidence of unfavorable (intermediate/high)-risk localized disease declined until 2014 before increasing between 2014 and 2015. Furthermore, there was a slow and gradual increase in the incidence of distant metastatic disease throughout the study period.
To the best of our knowledge, this is the first study to report on population-based incidence trends in localized (AJCC 7th edition N0M0) prostate cancer and across the NCCN risk groups used to guide clinical management. Prior studies were not able to benefit from the recent inclusion of validated and quality-assured PSA data in SEER and therefore have been limited to describing trends in SEER summary stage, which is not used in clinical practice, ${ }^{13,14}$ or in AJCC M1 disease, which cannot describe localized risk group patterns. ${ }^{15}$ By examining incidence rates according to NCCN risk group, these findings are not only novel and clinically relevant, they are also a more accurate reflection of the impact of PSA screening recommendations based on the natural history of prostate cancer. Specifically, because $65.7 \%$ of all localized disease and $94.0 \%$ of NCCN low-risk disease were PSA screen-detected from 2010 to 2011 in SEER (unpublished data), USPSTF recommendations against screening would be expected to have an immediate impact on localized disease incidence, with the 
$A_{1.1}$

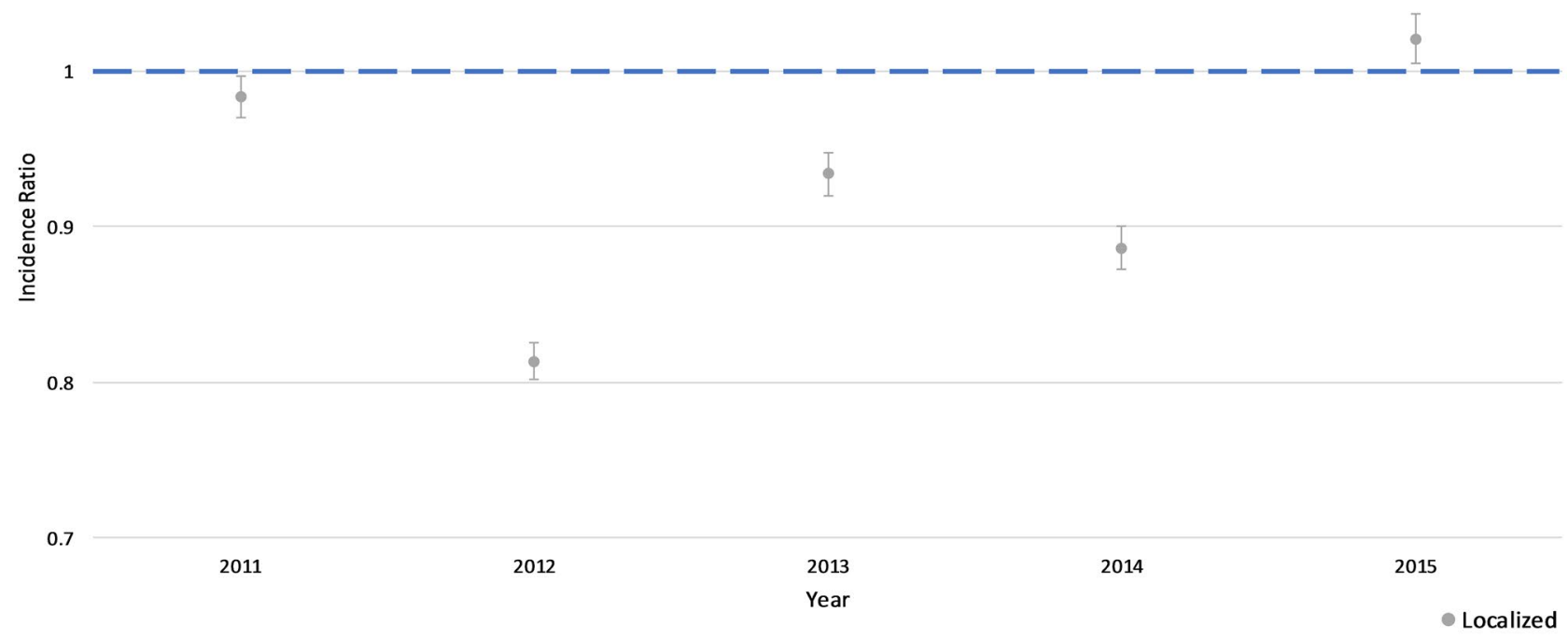

B 1.1

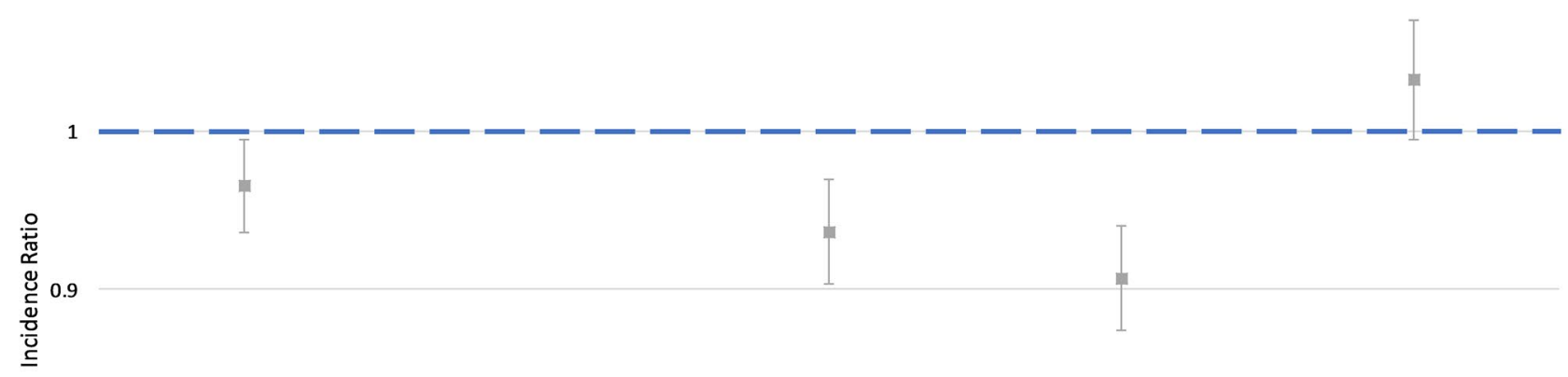

0.8

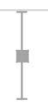

0.7

2011

2012

2013

2014

2015

Year

- Localized

Figure 2. Incidence ratios comparing consecutive annual age-adjusted incidence rates (with error bars representing $95 \%$ Cls) of prostate cancer diagnoses in the SEER*Stat U.S. Database stratified by age group ( $\geq 75$ vs. $50-74$ years of age) for (A, B) localized disease (NOMO), (C, D) metastatic disease (M1), (E, F) National Comprehensive Cancer Network (NCCN) low-risk localized disease, and (G, H) NCCN unfavorable (intermediate/high)-risk localized disease. Error bars represent 95\% Cls. Incidence ratios compare consecutive year-by-year baselines (2011 vs 2010, 2012 vs 2011, 2013 vs 2012, 2014 vs 2013, 2015 vs 2014).

greatest impact on NCCN low-risk localized disease-as was demonstrated in this study-presumably through the decreased detection of indolent and asymptomatic disease. The declines in low-risk disease were a goal of USPSTF recommendations against screening. However, many men with potentially curable disease may present with more advanced and difficult-to-cure disease later on, as suggested by the slow increases in metastatic disease and increasing incidence of unfavorable-risk disease between 2014 and 2015 . 
$\mathbf{C}_{1.3}$

1.2

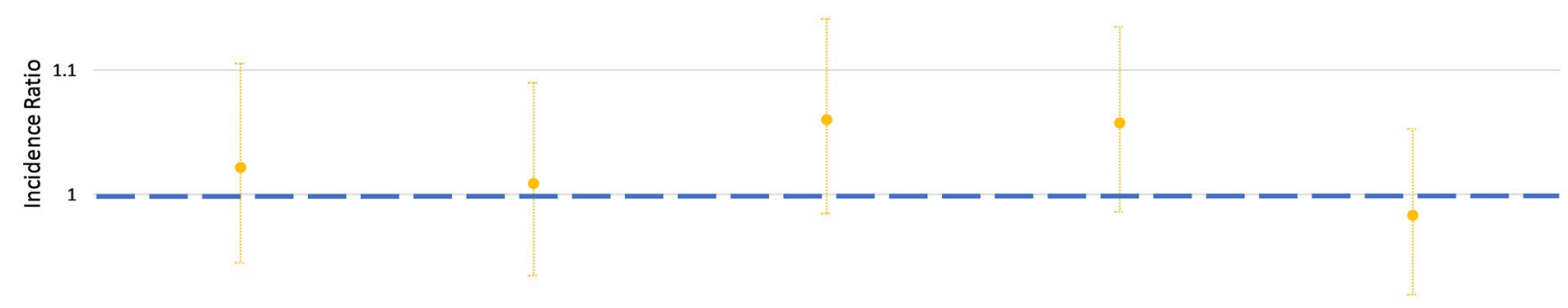

0.9

0.8

20112012

2013

2014

2015

Year

- Metastatic

$\mathbf{D}_{1.3}$

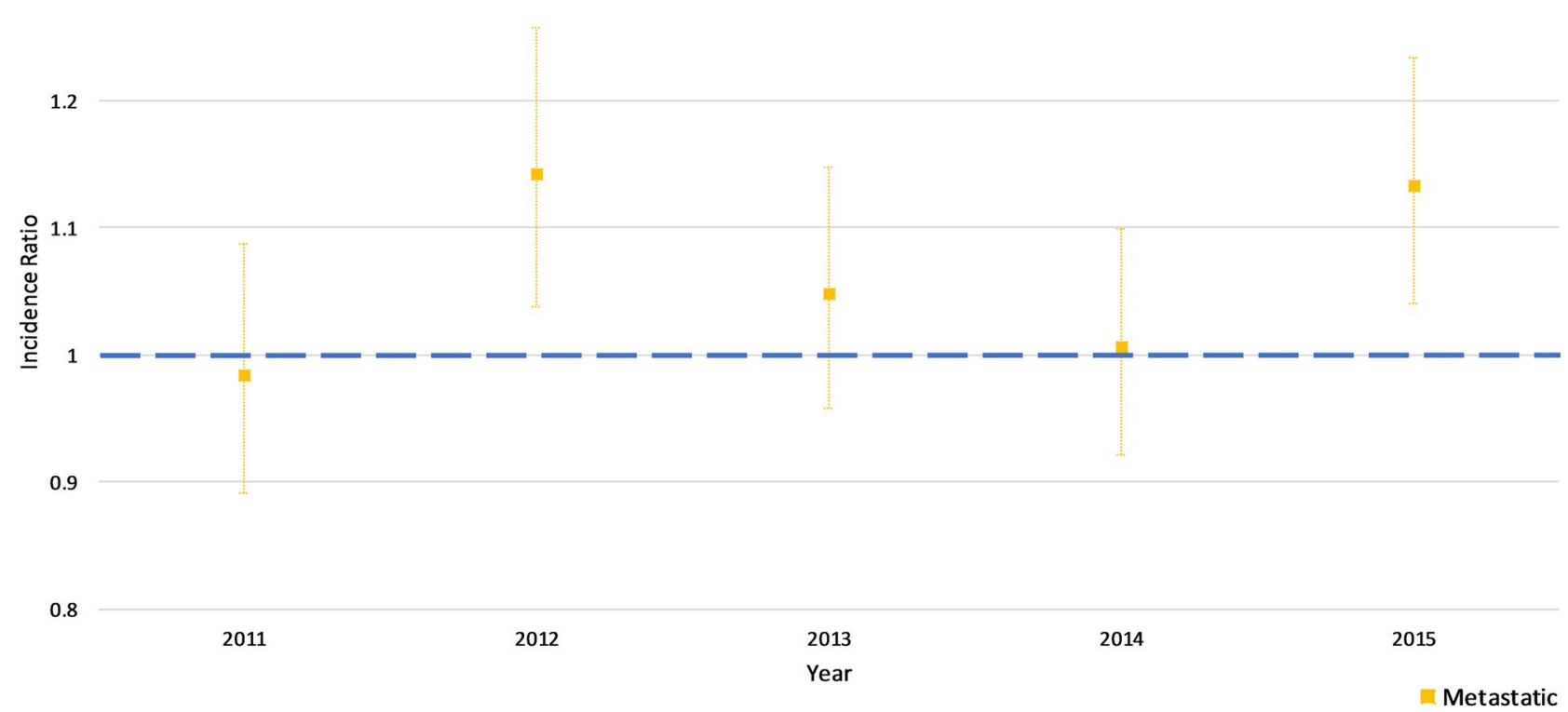

Figure 2. (Continued).

Notably, $\mathrm{Hu}$ et $\mathrm{al}^{15}$ demonstrated that incidence rates of distant metastatic disease among men aged $\geq 75$ years only increased by less than 1 per 100,000 persons from 2007 to 2013 (after USPSTF recommendations against PSA screening for men aged $\geq 75$ years in 2008), while we demonstrate that these rates increased by 5.8 per 100,000 from 2010 to 2015 . This is likely because population increases in de novo metastases may not be expected until $\geq 6$ years after development of disease in nonscreened individuals based on the natural history of prostate cancer. ${ }^{16-18}$ As such, the nonsignificant increases in metastatic disease (by $\sim 1$ case per 100,000 persons per year) for men aged $50-74$ years between 2010 and 2015 would be expected to similarly 
$E_{1.2}$

1.1

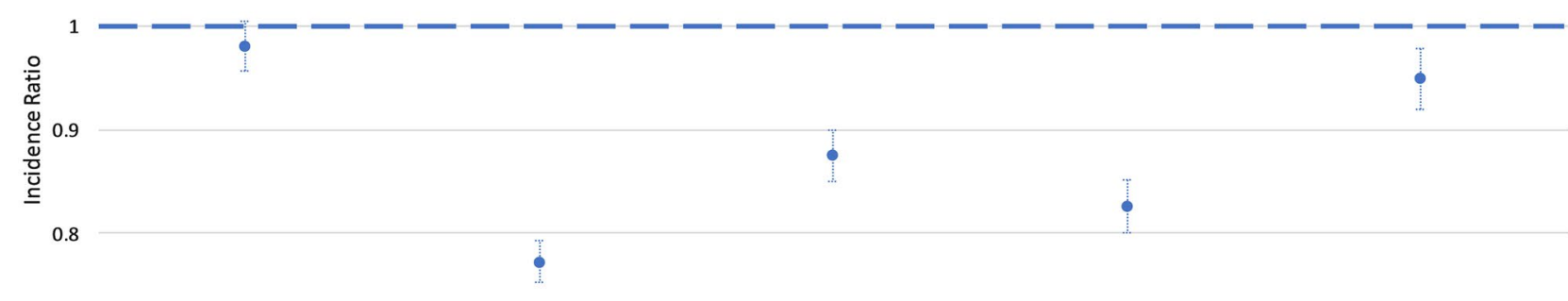

0.7

0.6

2012

2014

2015

Year

- Low-Risk

$\mathbf{F}_{1.2}$

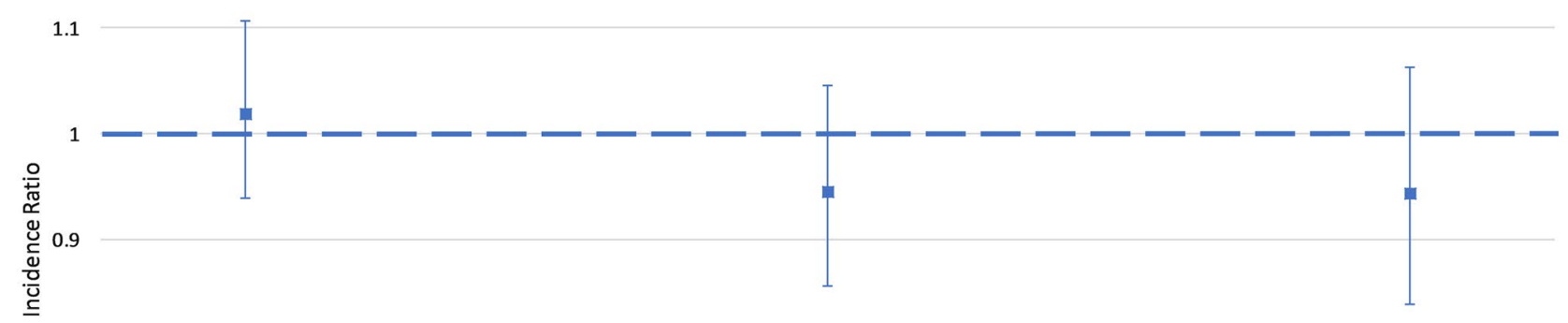

0.8

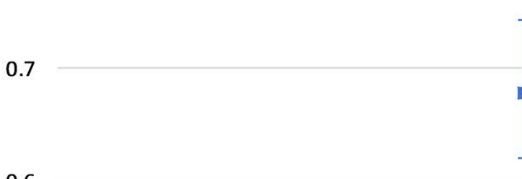

0.6

2011

2012

2013

Year

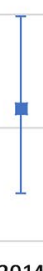

2014

2015

[ Low-Risk

Figure 2. (Continued).

continue increasing through 2018. Regardless, prostate cancer is a heterogenous disease, and the missed screening of higher-risk cancers, which could progress more quickly to symptomatic presentation, may lead to increases in metastases at earlier time points at the population level. The public health ramifications of a trend toward excess metastatic prostate cancer incidence would likely include not only greater disease morbidity (eg, metastatic bone pain, skeletal-related events) and prostate cancer mortality, but also greater treatmentrelated toxicity-most notably from lifelong hormonal therapy-and higher overall health care costs.

Additionally, our study demonstrated that the largest decrease in localized prostate cancer incidence occurred between 2011 and 2012_-suggesting that there may have been a decline in PSA screening throughout the year of 2012 
$\mathbf{G}_{1.2}$

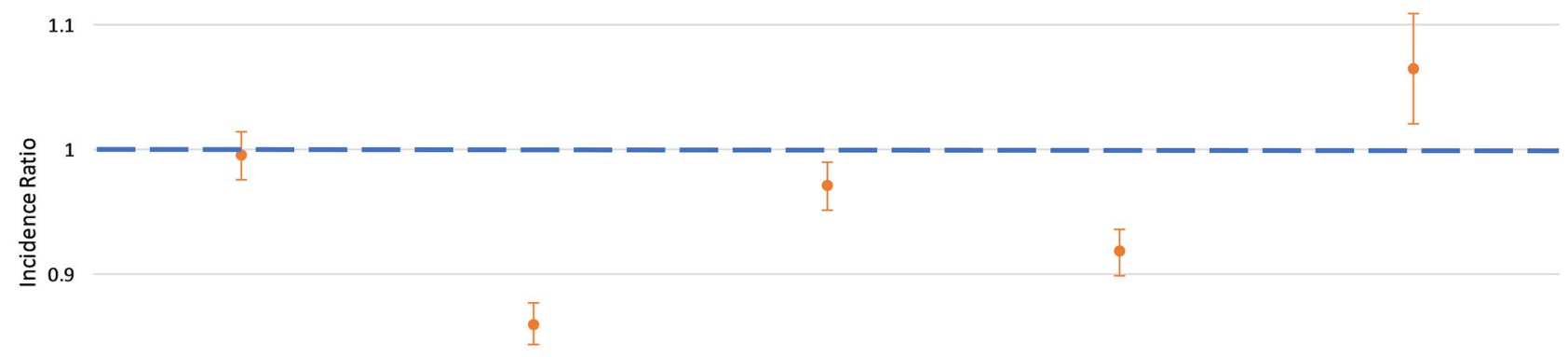

0.8

0.7

$20112012 \quad 2013$

2014

2015

Year

- Intermediate/High-Risk

$\mathbf{H}_{1.2}$

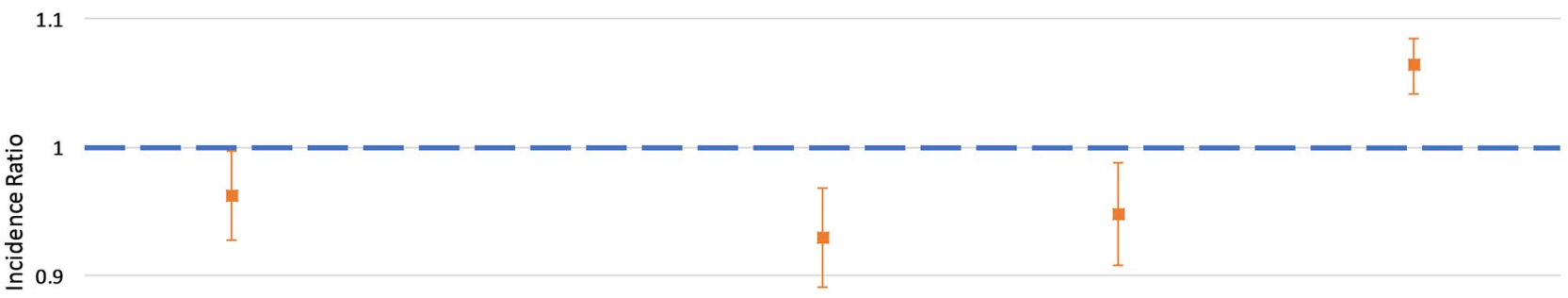

0.8

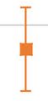

0.7

2011

2012

2013

2014

2015

Year

Intermediate/High-Risk

Figure 2. (Continued).

after the highly publicized draft recommendations were made public in October 2011.

Overall, our results demonstrate disease "reverse migration" away from indolent disease and toward more aggressive presentation following 2011-2012 USPSTF Grade D recommendations against PSA screening. Additional factors that may have contributed to these trends include changes in perceptions of PSA screening risks and benefits, concern of prostate cancer overdiagnosis or overtreatment, and emphasis on informed decision-making. Patients with unknown clinical information on NCCN risk factors (ie, PSA, Gleason score, T stage) were not included in analyses stratified by NCCN risk group, which could underestimate overall absolute incidence rates within risk groups; notably, there was no difference in the completeness of data across the study 
period, hence there is unlikely to be bias within the reported trends in relative ratios across years. This study was also limited by lack of SEER data on regional differences in incidence. Lastly, this study was limited by short follow-up, and further studies will be required to assess the long-term impact of recommendations on prostate cancer incidence and mortality. Furthermore, future studies will need to determine whether 2018 USPSTF Grade $\mathrm{C}$ recommendations again shift the prostate cancer landscape.

In conclusion, this report illustrates recent prostate cancer "reverse migration" away from indolent disease and toward more aggressive disease beginning in 2012. The incidence of localized disease declined across age groups from 2012 to 2015, with the greatest relative declines occurring in low-risk disease. Additionally, the incidence of distant metastatic disease gradually increased throughout the study period.

\section{FUNDING SUPPORT}

Timothy R. Rebbeck was funded by Health and Human Services grant CA184734. Brandon A. Mahal was funded by the PCF-ASTRO Award to End Prostate Cancer. Paul L. Nguyen was funded by the Prostate Cancer Foundation.

\section{CONFLICT OF INTEREST DISCLOSURES}

Shuang G. Zhao has received research funding from Genome Dx and has patents pending with Celgene and Decipher Biosciences. Paul L. Nguyen has received consulting fees from Nanobiotix, Bayer, Cota, Astellas, Ferring, Dendreon, Blue Earth Diagnostics, Genome Dx, Augmenix, Janssen; has received research funding from Janssen, Astellas, and the Prostate Cancer Foundation; and holds private equity with Augmenix. Brandon Mahal has received research grants from the Prostate Cancer Foundation and ASTRO. The other authors made no disclosures.

\section{AUTHOR CONTRIBUTIONS}

Santino S. Butler: data access, data analysis, responsibility for data, study concept and design, statistical analysis. Vinayak Muralidhar: data acquisition, data analysis, interpretation of data, writing-original draft, writingreview and editing. Shuang G. Zhao: data acquisition, data analysis, interpretation of data, writing-original draft, writing-review and editing. Nina N. Sanford: data acquisition, data analysis, interpretation of data, writing-original draft, writing-review and editing. Idalid Franco: data acquisition, data analysis, interpretation of data, writing-original draft, writingreview and editing. Zoe H. Fullerton: data acquisition, data analysis, interpretation of data, writing-original draft, writing-review and editing. Janice Chavez: data acquisition, data analysis, interpretation of data, writingoriginal draft, writing-review and editing. Anthony V. D'Amico: data acquisition, data analysis, interpretation of data, writing-original draft, writing-review and editing. Felix Y. Feng: data acquisition, data analysis, interpretation of data, writing-original draft, writing-review and editing. Timothy R. Rebbeck: data acquisition, data analysis, interpretation of data, writing-original draft, writing-review and editing. Paul L. Nguyen: data acquisition, data analysis, interpretation of data, writing-original draft, writing-review and editing. Brandon A. Mahal: data access, data analysis, responsibility for data, study concept and design, statistical analysis.

\section{REFERENCES}

1. Potosky AL, Miller BA, Albertsen PC, Kramer BS. The role of increasing detection in the rising incidence of prostate cancer. JAMA. 1995;273:548-552.

2. US Preventive Services Task Force. Screening for prostate cancer: U.S. Preventive Services Task Force recommendation statement. Ann Intern Med. 2008;149:185-191.

3. Moyer VA, et al. Screening for prostate cancer: U.S. Preventive Services Task Force recommendation statement. Ann Intern Med. 2012;157:120-134.

4. Andriole GL, Crawford ED, Grubb RL 3rd, et al. Mortality results from a randomized prostate-cancer screening trial. $N$ Engl J Med. 2009;360:1310-1319.

5. Schroder FH, Hugosson J, Roobol MJ, et al. Screening and prostatecancer mortality in a randomized European study. $N$ Engl J Med. 2009;360:1320-1328.

6. Penson DF. The pendulum of prostate cancer screening. JAMA. 2015;314:2031-2033.

7. Schroder FH, Hugosson J, Roobol MJ, et al. Screening and prostate cancer mortality: results of the European Randomised Study of Screening for Prostate Cancer (ERSPC) at 13 years of follow-up. Lancet. 2014;384:2027-2035.

8. Grossman DC, Curry SJ, Owens DK, et al. Screening for prostate cancer: US Preventive Services Task Force Recommendation Statement. JAMA. 2018;319:1901-1913.

9. NCCN Clinical Practice Guidelines in Oncology (NCCN Guidelines) Prostate Cancer. Version 2.2018. National Comprehensive Cancer Network, Inc. Published March 8, 2018. Accessed July 1, 2018. https:// www.nccn.org/professionals/physician_gls/pdf/prostate.pdf.

10. National Cancer Institute Surveillance, Epidemiology, and End Results Program. SEER*Stat Databases: November 2017 submission. Accessed July 1, 2018. https://seer.cancer.gov/data-software/documentation/ seerstat/nov2018/.

11. National Cancer Institute Surveillance, Epidemiology, and End Results Program. PSA values and SEER data. Accessed July 1, 2018. https:// seer.cancer.gov/data/psa-values.html.

12. Tiwari RC, Clegg LX, Zou Z. Efficient interval estimation for age-adjusted cancer rates. Stat Methods Med Res. 2006;15:547-569. doi: $10.1177 / 0962280206070621$

13. Jemal A, Fedewa SA, Ma J, et al. Prostate cancer incidence and PSA testing patterns in relation to USPSTF screening recommendations. JAMA. 2015;314:2054-2061. doi:10.1001/jama.2015.14905

14. Jemal A, Ma J, Siegel R, Fedewa S, Brawley O, Ward EM. Prostate cancer incidence rates 2 years after the US Preventive Services Task Force recommendations against screening. JAMA Oncol. 2016;2:1657-1660. doi:10.1001/jamaoncol.2016.2667

15. Hu JC, Nguyen P, Mao J, et al. Increase in prostate cancer distant metastases at diagnosis in the United States. JAMA Oncol. 2017;3: 705-707. doi:10.1001/jamaoncol.2016.5465

16. Draisma G, Boer R, Otto SJ, et al. Lead times and overdetection due to prostate-specific antigen screening: estimates from the European Randomized Study of Screening for Prostate Cancer. J Natl Cancer Inst. 2003;95:868-878.

17. Hugosson J, Aus G, Becker C, et al. Would prostate cancer detected by screening with prostate-specific antigen develop into clinical cancer if left undiagnosed? A comparison of two population-based studies in Sweden. BJU Int. 2000;85:1078-1084.

18. Tornblom M, Eriksson H, Franzen S, et al. Lead time associated with screening for prostate cancer. Int J Cancer. 2004;108:122-129. doi:10.1002/ijc. 11554 